\title{
Spontaneous tumour lysis syndrome
}

\author{
Natasha Kekre MD, Bojana Djordjevic MD, Claire Touchie MD
}

A 76-year-old man presented to the emergency department with a three-week history of nausea, vomiting, diarrhea and decreased appetite. He had lost $13.6 \mathrm{~kg}$ during this period. He denied any fevers or sweats. He had a left shoulder replacement three months before presentation and subsequently noticed decreased energy. He had also experienced mild epigastric pain during the 24 hours before presenting to hospital.

His medical history included hemochromatosis complicated by arthritis and diabetes requiring insulin treatment. His last phlebotomy was three years earlier. Regular follow-up with iron studies did not indicate the need for further phlebotomies. His history also included class III chronic kidney disease, hypertension, dyslipidemia and erectile dysfunction. He had a 30 pack-year smoking history and had quit 12 years earlier. His medications included acitretin, omeprazole, amlodipine, celecoxib, candesartan, levemir, salbutamol, indapamide, atorvastatin and testosterone.

Physical examination revealed stable vital signs, flat jugular venous pressure and hepatomegaly. The patient did not have any palpable lymphadenopathy or splenomegaly. The rest of his examination was unremarkable.

We suspected that the patient might be in a hyperglycemic hyperosmolar state because his initial glucometer reading was high (indicating a blood glucose level above $30 \mathrm{mmol} / \mathrm{L}$ ). Further investigations including serum calcium, phosphate, magnesium, liver enzymes and renal function tests were completed. The results of these tests (Table 1) were not consistent with our presumptive diagnosis of a hyperglycemic hyperosmolar state. The most impressive findings were the severely elevated lactate dehydrogenase level, hypocalcemia, hyperphosphatemia and hyperkalemia. This was quickly recognized as tissue breakdown, possibly spontaneous tumour lysis syndrome.

We initially gave the patient intravenous fluids and insulin, and calcium gluconate was added for the hyperkalemia. Because the laboratory findings were suggestive of possible tumour lysis syndrome, the patient was started on allo- purinol. Computed tomography of his abdomen showed a large hepatic mass (Figure 1). Over the next few hours, asterixis, confusion and anuria with persistent hyperkalemia developed. The patient underwent dialysis without any fluid removal. Two hours after dialysis, profound shock requiring vasopressors developed. He was transferred to the intensive care unit but died within hours.

The autopsy showed a tumour measuring $19.2 \times 11.0 \times 8.0 \mathrm{~cm}$ in the right hepatic lobe occupying about $40 \%$ of the total liver volume and with extension into the portal vein (Figure 2, Appendix 1, available at www.cmaj.ca/lookup /suppl/doi:10.1503/cmaj.111251/-/DC1). On microscopic examination, the tumour was a moderately differentiated hepatocellular carcinoma with extensive necrosis (Figure 3, Appendix 2, available at www.cmaj.ca/lookup/suppl/doi:10 .1503/cmaj.111251/-/DC1). The background liver contained micronodular steatosis as well as focal bridging fibrosis. There was no evidence of iron deposition in the liver.

The kidneys showed features consistent with diabetic nephropathy. Evaluation for acute tubular injury was not possible because of substantial artifacts from autolysis.

\section{Discussion}

Tumour lysis syndrome is a collection of metabolic and electrolyte disturbances that can be observed during the destruction of tumour cells. High cellular proliferation and turnover result in

\section{KEY POINTS}

- Tumour lysis syndrome occurs most commonly following treatment of a malignancy; however, it can occur spontaneously and must be recognized early to start appropriate treatment.

- This diagnosis should be considered if the electrolyte criteria for the syndrome are met, even in the absence of a previous diagnosis of malignant disease.

- Risk factors include bulky disease and high sensitivity to chemotherapy of a tumour, and renal impairment.

- Untreated hepatocellular carcinoma can lead to tumour lysis. 
hyperphosphatemia, hypocalcemia, hyperkalemia and hyperuricemia. The often fatal consequences of the release of the contents of tumour cells include seizure, acute kidney injury and cardiac arrhythmia.'

\section{Table 1: Results of laboratory investigations}

\begin{tabular}{|c|c|c|c|}
\hline Description & Result & Units & Reference range \\
\hline Leukocyte count & 13.6 & $10^{9} / \mathrm{L}$ & $3.0-10.5$ \\
\hline Hemoglobin & 121 & $g / L$ & $130-170$ \\
\hline Hematocrit & 0.391 & & $0.38-0.50$ \\
\hline $\begin{array}{l}\text { Mean corpuscular } \\
\text { volume }\end{array}$ & 102.5 & $\mathrm{fL}$ & $80.0-100.0$ \\
\hline Platelets & 194 & $10^{9} / \mathrm{L}$ & $125-400$ \\
\hline Neutrophils & 11 & $10^{9} / \mathrm{L}$ & $2.0-7.5$ \\
\hline Lymphocytes & 1.4 & $10^{9} / \mathrm{L}$ & $1.0-4.0$ \\
\hline Monocytes & 1.1 & $10^{9} / \mathrm{L}$ & $0.1-1.0$ \\
\hline Eosinophils & 0.1 & $10^{9} / \mathrm{L}$ & $0.0-0.5$ \\
\hline Basophils & 0 & $10^{9} / \mathrm{L}$ & $0.0-0.1$ \\
\hline Sodium & 132 & $\mathrm{mmol} / \mathrm{L}$ & $136-145$ \\
\hline Potassium & 6.9 & $\mathrm{mmol} / \mathrm{L}$ & $3.5-5.1$ \\
\hline Chloride & 98 & $\mathrm{mmol} / \mathrm{L}$ & $98-107$ \\
\hline Carbon dioxide & 17 & $\mathrm{mmol} / \mathrm{L}$ & $21-32$ \\
\hline Anion gap & 17 & $\mathrm{mmol} / \mathrm{L}$ & $5-12$ \\
\hline Glucose & 24.5 & $\mathrm{mmol} / \mathrm{L}$ & $3.8-11$ \\
\hline Urea & 37.4 & $\mathrm{mmol} / \mathrm{L}$ & $2.1-8.0$ \\
\hline Creatinine & 363 & $\mu \mathrm{mol} / \mathrm{L}$ & $62-106$ \\
\hline Ketones & Negative & & \\
\hline Phosphorus & 2.97 & $\mathrm{mmol} / \mathrm{L}$ & $0.81-1.58$ \\
\hline Calcium & 1.93 & $\mathrm{mmol} / \mathrm{L}$ & $2.20-2.52$ \\
\hline Magnesium & 1.07 & $\mathrm{mmol} / \mathrm{L}$ & $0.74-1.03$ \\
\hline Albumin & 30 & $g / L$ & $34-46$ \\
\hline Total bilirubin & 22 & $\mu \mathrm{mol} / \mathrm{L}$ & $3-17$ \\
\hline $\begin{array}{l}\text { Alanine } \\
\text { aminotransferase }\end{array}$ & 387 & $\mathrm{U} / \mathrm{L}$ & $17-63$ \\
\hline $\begin{array}{l}\text { Aspartate } \\
\text { aminotransferase }\end{array}$ & 8284 & $\mathrm{U} / \mathrm{L}$ & $15-37$ \\
\hline $\begin{array}{l}\text { Gamma- } \\
\text { glutamyltransferase }\end{array}$ & 444 & $\mathrm{U} / \mathrm{L}$ & $15-85$ \\
\hline Alkaline phosphatase & 170 & $\mathrm{U} / \mathrm{L}$ & $50-136$ \\
\hline $\begin{array}{l}\text { Lactate } \\
\text { dehydrogenase }\end{array}$ & $>6000$ & $\mathrm{U} / \mathrm{L}$ & $100-190$ \\
\hline Creatine kinase & 704 & $\mathrm{U} / \mathrm{L}$ & $20-215$ \\
\hline Osmolality & 346 & $\mathrm{mmol} / \mathrm{k}$ & 280-295 \\
\hline Troponin I & 0.28 & $\mu \mathrm{g} / \mathrm{L}$ & $<0.04$ \\
\hline Uric acid & 971 & $\mu \mathrm{mol} / \mathrm{L}$ & $208-428$ \\
\hline Lactic acid & 2.8 & $\mathrm{mmol} / \mathrm{L}$ & $0.5-2.2$ \\
\hline \multicolumn{4}{|l|}{ Venous gas } \\
\hline $\mathrm{pH}$ & 7.27 & & 7.31-7.41 \\
\hline $\mathrm{PCO}_{2}$ & 36 & $\mathrm{~mm} \mathrm{Hg}$ & $41-51$ \\
\hline $\mathrm{HCO}_{3}$ & 17 & $\mathrm{mmol} / \mathrm{L}$ & $18-23$ \\
\hline
\end{tabular}

\section{Diagnosis}

Cairo and Bishop proposed laboratory and clinical criteria (Box 1) for tumour lysis syndrome. ${ }^{2}$ To fulfill the diagnosis, at least two laboratory criteria must be present for three days before treatment or up to seven days after treatment. A clinical diagnosis consists of one clinical symptom in addition to two laboratory criteria. Our patient met the clinical diagnosis of tumour lysis syndrome based on his hyperphosphatemia, hyperuricemia, hyperkalemia and acute renal failure.

\section{Risk stratification}

Tumour lysis syndrome is well established in association with hematologic malignant diseases, specifically acute leukemia and non-Hodgkin lymphoma. ${ }^{3}$ The incidence of tumour lysis in the treatment of hematologic malignant disease is between $4 \%-42 \%,{ }^{3}$ although the incidence appears to be decreasing with the use of preventive measures. ${ }^{4}$ Although it is more commonly seen after treatment, the spontaneous form of this syndrome has been reported mostly among patients with hematologic malignant diseases. ${ }^{5}$ The prevalence of spontaneous tumour lysis in patients with solid tumours is harder to ascertain because it is based primarily on case reports.

Most guidelines indicate that the risk of tumour lysis is based on two factors. ${ }^{3,46}$ First, the risk is related to the malignancy itself. Increased size of the cancer mass and involvement of other organs and bone marrow increase the risk. The cell lysis potential of the cancer, including the proliferative ability and chemosensitivity of the tumour, also poses a risk for tumour lysis. For example, highly proliferative malignant diseases, such as Burkitt lymphoma and acute lymphoblastic lymphoma, are associated with a high risk of tumour lysis. Second, patient factors such as dehydration and renal failure make the clearance of intracellular metabolites more difficult, thus increasing the risk of tumour lysis.

An expert consensus panel has recommended that patients with acute leukemia and aggressive lymphomas, such as Burkitt lymphoma, be considered at least at intermediate risk for tumour lysis syndrome. ${ }^{6}$ These patients can be classified as high risk if they have bulky disease, a highly elevated leukocyte count on presentation or renal involvement. In a retrospective analysis involving patients with proven hematologic malignant disease and acute renal failure, the rate of spontaneous tumour lysis syndrome was $1.08 \%$. $^{?}$

The consensus guidelines indicate that all solid tumours are low risk for lysis, unless the disease is bulky or highly sensitive to chemotherapy, in which case the tumour should be classified as intermediate risk. If the patient has risk factors 
similar to those of other hematologic malignancies, including chronic renal insufficiency, oliguria, dehydration or hypotension, the patient may potentially be at high risk of this syndrome.

Evidence of tumour lysis syndrome in solid tumours is restricted to case reports and small case series because the risk of this syndrome in

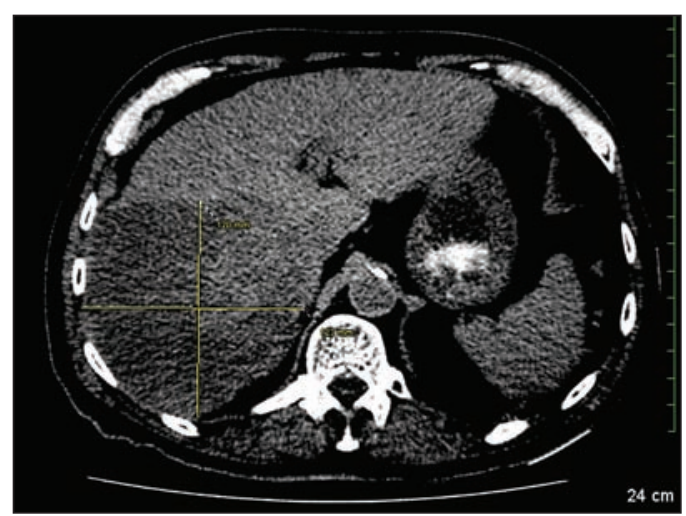

Figure 1: Large lobulated mass occupying the right lobe of the liver. The yellow cross indicates the largest tumour (123 mm diameter).

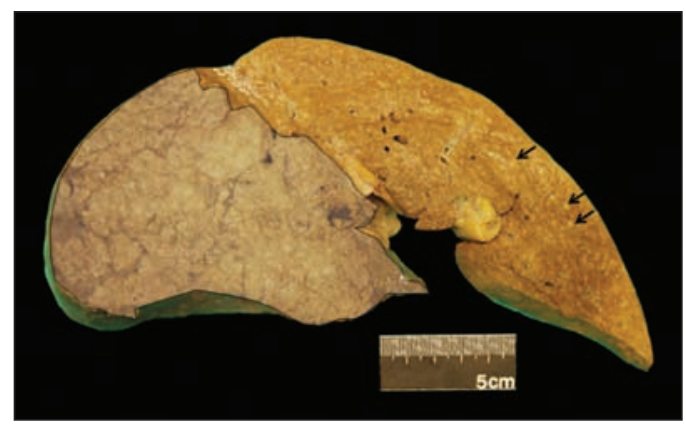

Figure 2: Coronal section of the liver. The main tumour mass $(19.2 \times 11.0 \times 8.0 \mathrm{~cm})$ involved most of the right hepatic lobe (outlined). Satellite nodules $(0.1-0.5 \mathrm{~cm})$ were present in the left lobe (arrows).

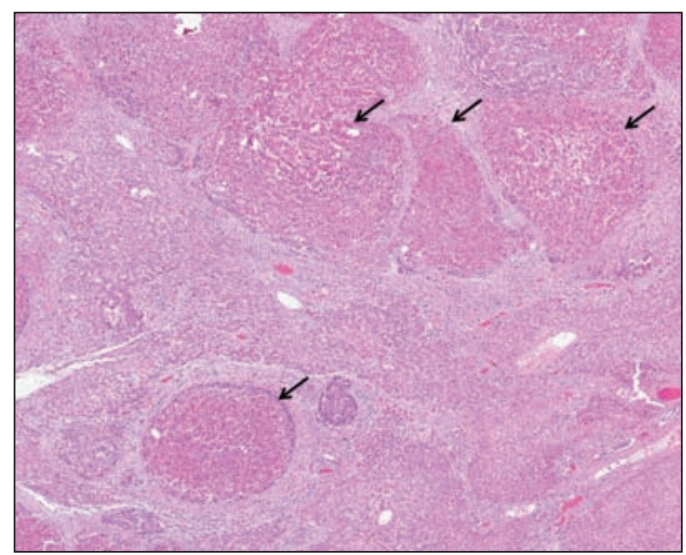

Figure 3: Microscopic image of the tumour. The tumour was a moderately differentiated hepatocellular carcinoma. It had extensive areas of necrosis (arrows) and diffusely infiltrated the liver parenchyma (hematoxylin and eosin stain, $2 \times$ magnification). these patients is generally low. ${ }^{6}$ There is, however, a growing number of reported cases of tumour lysis syndrome in such patients after the initiation of treatment, including corticosteroids, radiation and chemotherapy. ${ }^{8-11}$ Although it occurs rarely, this syndrome can also be spontaneous in patients with solid tumours, including, but not limited to, gastric, lung and breast cancer. ${ }^{12-15}$

There is limited information about tumour lysis syndrome and malignant involvement of the liver. Tumour lysis has been documented in patients with hepatocellular carcinoma after treatment with sorafenib ${ }^{16,17}$ and transarterial chemoembolization. ${ }^{18,19}$ One case report identified spontaneous tumour lysis syndrome in hepatocellular carcinoma, specifically in a patient who did not receive chemotherapy because of advanced stage of disease. ${ }^{12}$

\section{Prevention}

The mainstay of prevention of tumour lysis syndrome consists of hydration. Intravenous hydration promotes the excretion of uric acid and other metabolites that lead to this syndrome. It is important to recognize dehydration as a risk factor so that prompt fluid resuscitation can be started. Allopurinol further prevents its development by inhibiting xanthine oxidase, the enzyme responsible for the formation of uric acid from xanthine. Consensus guidelines recommend that patients at low and intermediate risk of tumour lysis syndrome should be monitored closely and treated with rehydration and allopurinol to prevent its development. ${ }^{6}$ Patients at high risk are recommended to receive rasburicase prophylactically because it is the only agent currently available to reduce the level of uric acid in the blood. ${ }^{6}$

\section{Management}

Hydration and rasburicase, which catalyzes the conversion of uric acid to a more stable metabolite that can be readily excreted, are equally important in the management of tumour lysis syndrome, with special attention to addressing

Box 1: Cairo-Bishop diagnostic criteria for tumour lysis syndrome Laboratory criteria

- Uric acid $\geq 476 \mu \mathrm{mol} / \mathrm{mL}$ or a $25 \%$ increase from baseline

- Phosphorus $\geq 1.45 \mathrm{mmol} / \mathrm{L}$ or a $25 \%$ increase from baseline

- Potassium $\geq 6.0 \mathrm{mmol} / \mathrm{L}$ or a $25 \%$ increase from baseline

- Corrected calcium $\leq 1.75 \mathrm{mmol} / \mathrm{L}$ or a $25 \%$ decrease from baseline

Clinical criteria*

- Creatinine $\geq 1.5$ times the upper limit of normal

- Cardiac arrhythmia or sudden death

- Seizure

*May not be directly related to a therapeutic agent. 
the potentially fatal electrolyte imbalances observed in the syndrome. Hyperkalemia should be monitored closely and treated with insulin, glucose and calcium gluconate, ${ }^{4}$ as per established protocols. ${ }^{20}$ Hemodialysis should be considered for refractory hyperkalemia, especially in the setting of oliguria. The rapid accumulation of potassium in the setting of oliguria and the potential for hypocalcemia from the hyperphosphatemia are two reasons why a lower threshold for starting dialysis in these patients has been suggested. ${ }^{4}$

Our patient underwent aggressive fluid resuscitation and was given insulin, glucose and calcium gluconate for his hyperkalemia. Unfortunately, oliguria and refractory hyperkalemia quickly developed, requiring dialysis. The patient's condition deteriorated shortly after dialysis, presumably from a large cytokine surge, and he ultimately died.

\section{Recognition and early treatment}

Tumour lysis syndrome is potentially fatal, thus it is imperative that clinicians recognize the constellation of laboratory and clinical findings that suggest the diagnosis. In initial studies, the incidence of life-threatening complications from tumour lysis syndrome was as high as $13 \%$ in patients with hematologic malignancies. ${ }^{3}$ Another study showed that the rate of death was one in three among patients who developed tumour lysis syndrome following treatment of a solid malignancy. ${ }^{21}$

The findings of the laboratory features of tumour lysis syndrome, including hyperuricemia, hyperphosphatemia, hyperkalemia and hypocalcemia, should prompt the search for an underlying malignancy. In our patient, the presence of hepatomegaly on examination, particularly in the context of suspected tumour lysis syndrome, was the impetus to order computed tomography of his abdomen, which revealed a large hepatic mass. Even without pathologic confirmation of underlying malignant disease, certain risk factors for tumour lysis syndrome, including bulky disease, dehydration and renal failure, can help to validate the diagnosis and ensure that adequate treatment is started early.

Our patient had many risk factors for tumour lysis syndrome, including bulky disease greater than $10 \mathrm{~cm}$ and pre-existing renal compromise. ${ }^{1}$ Extensive tumour necrosis, as seen on autopsy, supported our clinical diagnosis. Although our patient's hemochromatosis was under good control clinically and as shown by the absence of iron deposits in the liver parenchyma, this condition may have been the factor that predisposed him to hepatocellular carcinoma. The bridging fibrosis seen in the background liver tissue on autopsy was in keeping with long-standing parenchymal injury.

Acute kidney injury and dramatic electrolyte imbalances should prompt consideration of spontaneous tumour lysis syndrome, even in the absence of a previous diagnosis of malignant disease. Quick initiation of appropriate treatment is essential.

\section{References}

1. Mughal TI, Ejaz AA, Foringer JR, et al. An integrated clinical approach for the identification, prevention, and treatment of tumor lysis syndrome. Cancer Treat Rev 2010;36:164-76.

2. Cairo MS, Bishop M. Tumour lysis syndrome: new therapeutic strategies and classification. Br J Haematol 2004;127:3-11.

3. Coiffier B, Altman A, Pui CH, et al. Guidelines for the management of pediatric and adult tumor lysis syndrome: an evidencebased review. J Clin Oncol 2008;26:2767-78.

4. Howard SC, Jones DP, Pui C. The tumor lysis syndrome. N Engl J Med 2011;364:1844-54.

5. Jasek AM, Day HJ. Acute spontaneous tumor lysis syndrome. Am J Hematol 1994;47:129-31.

6. Cairo MS, Coiffier B, Reiter A, et al. TLS Expert Panel. Recommendations for the evaluation of risk and prophylaxis of tumour lysis syndrome (TLS) in adults and children with malignant diseases: an expert TLS panel consensus. Br J Haematol 2010;149: 578-86.

7. Hsu HH, Chan YL, Huang CC. Acute spontaneous tumor lysis presenting with hyperuricemic acute renal failure: clinical features and therapeutic approach. J Nephrol 2004;17:50-6.

8. Lin CJ, Lim KH, Cheng YC, et al. Tumor lysis syndrome after treatment with gemcitabine for metastatic transitional cell carcinoma. Med Oncol 2007;24:455-7.

9. Wright JL, Lin DW, Dewan P, et al. Tumor lysis syndrome in a patient with metastatic, androgen independent prostate cancer. Int J Urol 2005;12:1012-3.

10. Habib GS, Saliba WR. Tumor lysis syndrome after hydrocortisone treatment in metastatic melanoma: a case report and review of the literature. Am J Med Sci 2002;323:155-7.

11. Rostom AY, El-Hussainy G, Kandil A, et al. Tumor lysis syndrome following hemi-body irradiation for metastatic breast cancer. Ann Oncol 2000;11:1349-51.

12. Vaisban E, Braester A, Mosenzon O, et al. Spontaneous tumor lysis syndrome in solid tumors: Really a rare condition? Am J Med Sci 2003;325:38-40.

13. Woo IS, Kim JS, Park MJ, et al. Spontaneous acute tumor lysis syndrome with advanced gastric cancer. J Korean Med Sci 2001; 16:115-8.

14. Feld J, Mehta H, Burkes RL. Acute spontaneous tumor lysis syndrome in adenocarcinoma of the lung: a case report. Am J Clin Oncol 2000;23:491-3.

15. Sklarin NT, Markham M. Spontaneous recurrent tumor lysis syndrome in breast cancer. Am J Clin Oncol 1995;18:71-3.

16. Huang WS, Yang CH. Sorafenib induced tumor lysis syndrome in an advanced hepatocellular carcinoma patient. World J Gastroenterol 2009;15:4464-6.

17. Shiozawa K, Watanabe M, Takenaka H, et al. Tumor lysis syndrome after sorafenib for hepatocellular carcinoma: a case report. Hepatogastroenterology 2010;57:688-90.

18. Hsieh PM, Hung KC, Chen YS. Tumor lysis syndrome after transarterial chemoembolization of hepatocellular carcinoma: case reports and literature review. World J Gastroenterol 2009; 15:4726-8.

19. Shiba H, Ishida Y, Wakiyama S, et al. Acute tumor lysis syndrome after transarterial chemoembolization for hepatocellular carcinoma. Cancer Sci 2008;99:2104-5.

20. Elliott MJ, Ronksley PE, Clase CM, et al. Management of patients with acute hyperkalemia. CMAJ 2010;182:1631-5.

21. Baeksgaard L, Sørensen JB. Acute tumor lysis syndrome in solid tumors - A case report and review of the literature. Cancer Chemother Pharmacol 2003;51:187-92.

Affiliations: From the Department of Medicine (Kekre, Touchie), Department of Pathology and Laboratory Medicine (Djordjevic), University of Ottawa, Ottawa, Ont.

Contributors: All of the authors were involved in drafting the article or revising it critically for important intellectual content, and all of the authors approved the final version submitted for publication. 